\title{
Flux-cancellation effect on narrow-channel magnetoresistance fluctuations
}

\author{
C. W. J. Beenakker and H. van Houten \\ Philips Research Laboratories, NL-5600 JA Eindhoven, The Netherlands
}

(Received 19 November 1987)

\begin{abstract}
The magnetic-field correlation function of the universal conductance fluctuations (UCF) in a laterally confined two-dimensional electron gas is calculated in the high-mobility regime. Boundary scattering induces cancellations of the magnetic flux enclosed by electron trajectories, which leads to an enhanced correlation field. The theory is discussed in relation to a recent observation of UCF in a narrow $\mathrm{GaAs}-\mathrm{Al}_{x} \mathrm{Ga}_{1-x} \mathrm{As}$ heterostructure.
\end{abstract}

An interesting development in the field of quantum transport in disordered metals is the Al'tshuler-Lee-Stone theory ${ }^{1,2}$ of universal conductance fluctuations (UCF). This theory predicts aperiodic fluctuations in the conductance as a function of magnetic field, with an rms amplitude at $T=0$ of order $e^{2} / \hbar$, independent of sample size or degree of disorder. Such UCF have been observed in metal wires and rings and in quasi-one-dimensional (1D) semiconductor devices. ${ }^{3}$ Their origin is the quantum interference of electrons on different trajectories, which surprisingly does not average out to zero within a few (elastic) collisions. (For a simple physical picture, see Ref. 4.) To destroy phase coherence, inelastic processes are necessary, but these become increasingly rare at low temperatures. An increment in magnetic-field shifts the phases of the electrons so that a different interference pattern results, and this is seen in the magnetoresistance fluctuations.

Recently, several groups ${ }^{5-10}$ have observed fluctuations in the perpendicular field magnetoresistance of laterally confined 2D electron gases in $\mathrm{GaAs}-\mathrm{Al}_{x} \mathrm{Ga}_{1-x}$ As heterostructures. Because of the high electron mobility in these quasi-1D channels, the elastic mean free path $l_{e}$ (associated with impurity scattering) can be much larger than the channel width $W$. This leads to an interesting modification of the current theory, in which the dirty metal limit $l_{e} \ll W$ is assumed. ${ }^{11}$ The effect we have in mind is the flux cancellation known from superconductivity, ${ }^{12}$ and studied recently ${ }^{13-15}$ in relation to the weak localization peak in the magnetoresistance. As illustrated in Fig. 1 , the intersecting trajectories of two electrons moving ballistically from one boundary to the other can enclose zero flux, due to a geometric cancellation. As a consequence, the electrons acquire no relative phase shift in a magnetic field. One would, therefore, expect the correlation field for magnetoresistance fluctuations to be enhanced in high-mobility channels with $l_{e}>W$. We note that finite-size enhancement of the correlation field was seen, and understood as a geometrical effect, in previous numerical work by Lee, Stone, and Fukuyama. " The calculation of the enhancement is the main issue of the present paper.

For simplicity, we limit ourselves here to the quasi-1D geometry of the experiments mentioned above ( $a$ thin metal film in parallel magnetic field is analogous). We consider the case $W \ll l_{\Phi} \ll L$, where $L$ is the length of the channel, and $l_{\phi}$ the phase-coherence length. There is an additional restriction, which is essential, that the motion along the channel is diffusive on the phase-coherence time scale, that is to say $\tau_{\phi} \gg \tau_{e}$. [We use the definitions $l_{\phi} \equiv\left(D \tau_{\phi}\right)^{1 / 2}, l_{e} \equiv v_{F} \tau_{e}$, with $D$ the diffusion coefficient and $v_{F}$ the Fermi velocity.] We will return to this restriction below. The quantity to be calculated is the correlation function 11,16

$$
F(\Delta B) \equiv\langle\delta G(B) \delta G(B+\Delta B)\rangle .
$$

Here $\delta G(B) \equiv G(B)-\langle G(B)\rangle$, with $G(B)$ the conductance in a magnetic field $B$ (the angle brackets denote an average over impurity configurations). Note that $F$ is $B$ independent, under the assumption that $B$ is outside the region of the weak localization peak in the magnetoresis-

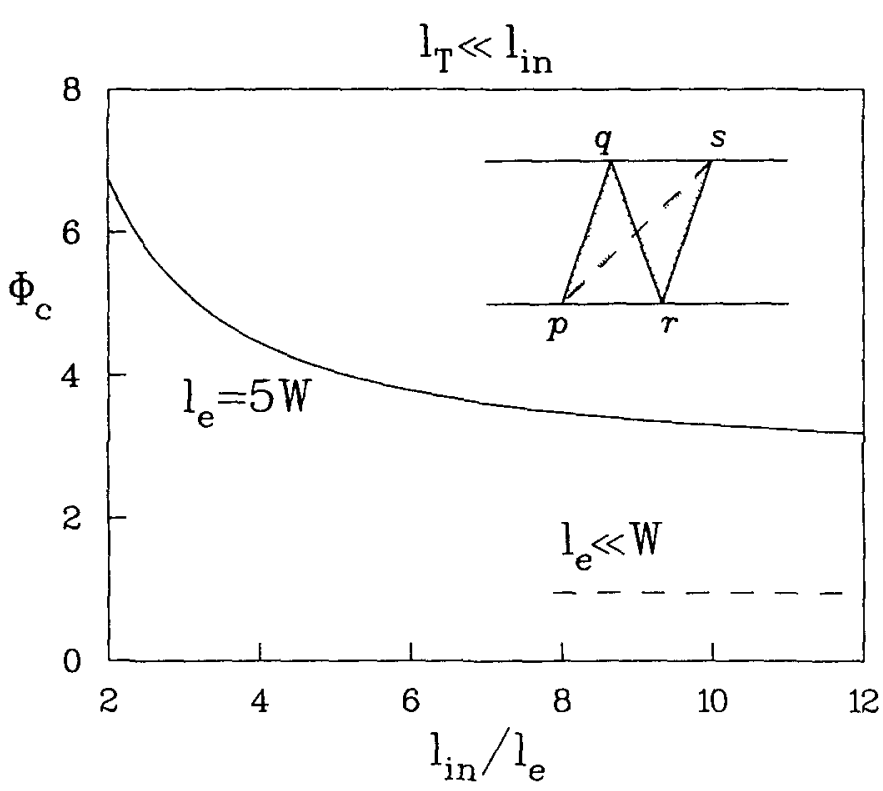

FIG. 1. Plot of the correlation flux $\Phi_{c}$ (in units of $2 \pi \hbar / e$ ) vs the zero-field phase-coherence length $l_{\mathrm{n}} \equiv l_{\phi}(0)$ (normalized by the elastic mean free path $l_{e}$ ). The solid curve is for a highmobility channel [Eq. (10)], the dashed line is the dirty metal value. The inset shows two trajectories of an electron in a narrow channel (dashed and solid lines) to illustrate the characteristic flux cancellation: The relative phase shift acquired in a perpendicular magnetic field is proportional to the flux enclosed by the contour pqrsp, which is zero. 
tance around zero field. (Then only the diffusion propagator contributes to $F$, which is sensitive to field differences $\Delta B$, not to $B$ itself. ${ }^{1,16}$ ) The value $F(0)$ equals the variance of the conductance fluctuations, and the field increment $\Delta B_{c}$ such that $F\left(\Delta B_{c}\right)=\frac{1}{2} F(0)$ is by definition the correlation field.

The variance is affected by boundary scattering only implicitly via the diffusion coefficient, so that no new analysis is required. At $\Delta B=0$ one has the two formulas ${ }^{11}$

$$
\begin{aligned}
& F=\alpha\left(\frac{e^{2}}{2 \pi \hbar}\right)^{2}\left(\frac{l_{\phi}}{L}\right)^{3} \text { if } l_{\phi} \ll l_{T}, \\
& F=\beta\left(\frac{e^{2}}{2 \pi \hbar}\right)^{2} \frac{l_{T}^{2} l_{\phi}}{L^{3}} \text { if } l_{\phi} \gg l_{T} .
\end{aligned}
$$

The thermal length is defined by $l_{T} \equiv\left(\hbar D / k_{B} T\right)^{1 / 2}$. The above asymptotic expressions are given in Ref. 11 up to unspecified numerical coefficients $\alpha$ and $\beta$. This has caused considerable uncertainties in the experimental literature. To eliminate these, we have evaluated the series and integrals of Lee et al. ${ }^{11}$ (which can be done analytically in the asymptotic regimes) and find $\alpha=6$, $\beta=\frac{4}{3} \pi$. For the analysis of experiments in which $l_{\phi}$ and $l_{T}$ are comparable, we also give a formula which interpolates between Eqs. (2a) and (2b):

$$
F \approx 6\left(\frac{e^{2}}{2 \pi \hbar}\right)^{2}\left(\frac{l_{\phi}}{L}\right)^{3}\left[1+\frac{9}{2 \pi}\left(\frac{l_{\phi}}{l_{T}}\right)^{2}\right]^{-1}
$$

This formula is approximate, but the differences with the full expressions of Lee et al. ${ }^{11}$ are not significant (less than $10 \%$ ).

The correlation field $\Delta B_{c}$ depends on the geometry of the trajectories (via the enclosed flux), and this is where boundary scattering comes in explicitly. The diagrammatic analysis of Lee et al. ${ }^{11}$ gives $F$ in terms of the diffusion propagator $P\left(\mathbf{r}, \mathbf{r}^{\prime} ; t\right)$. This quantity is the product of three terms: ${ }^{17}$ (1) the classical probability to diffuse from $\mathbf{r}$ to $\mathbf{r}^{\prime}$ in a time $t$ (independent of $B$ in the field range of interest); (2) the relaxation factor $\exp \left[-t / \tau_{\phi}(0)\right]$, with $\tau_{\phi}(0)$ the zero-field phase-coherence time (also referred to in the literature as the inelastic scattering time $\left.\tau_{\text {in }}\right)$; (3) the average phase factor $\left\langle e^{i \Delta \phi}\right\rangle$, with $\Delta \phi$ the phase shift induced by the field increment $\Delta B$. More explicitly,

$$
\begin{aligned}
\left\langle e^{i \Delta \phi}\right\rangle & \equiv\left\langle\exp \left(\frac{i e}{\hbar} \int_{\mathrm{r}}^{\mathrm{r}^{\prime}} \Delta \mathbf{A} \cdot d l\right)\right\rangle \\
& =\exp \left(-t / \tau_{\Delta B}\right)
\end{aligned}
$$

where the line integral of the vector potential increment
$\Delta \mathbf{A}$ is along a classical trajectory which goes from $\mathbf{r}$ to $\mathbf{r}^{\prime}$ in a time $t$, and the average is taken over all such trajectories. ${ }^{18}$ As a consequence of Eq. (4), proven in Ref. 14, the effect of a nonzero $\Delta B$ on the diffusion propagator is simply to increase its relaxation rate by an amount $1 / \tau_{\Delta B}$,

$$
1 / \tau_{\phi}(\Delta B)=1 / \tau_{\phi}(0)+1 / \tau_{\Delta B} .
$$

It follows that the correlation function $F(\Delta B)$ is given by Eqs. (2) and (3), with a $\Delta B$-dependent length $l_{\phi}(\Delta B) \equiv\left[D \tau_{\phi}(\Delta B)\right]^{1 / 2}$. By solving $F\left(\Delta B_{c}\right)=\frac{1}{2} F(0)$ we then find that the correlation field is determined by the relation

$$
\tau_{\Delta B_{c}}=\gamma \tau_{\phi}(0),
$$

where $\gamma=\frac{1}{3}$ if $l_{\phi} \gg l_{T}$, and $\gamma=1 /\left(4^{1 / 3}-1\right) \approx 1.7$ if $l_{\phi} \ll l_{T}$. [For comparable $l_{T}$ and $l_{\phi}$ the value of $\tau_{\Delta B_{c}}$ can be obtained using the interpolation formula (3).]

It remains to determine $\tau_{\Delta B}$. In Ref. 14, the relaxation time $\tau_{B}$ relevant to weak localization in a magnetic field $B$ was calculated for the present geometry. The relaxation time $\tau_{\Delta B}$ relevant to UCF is obtained from those results simply by replacing $B$ by $\frac{1}{2} \Delta B$ [compare the phase definitions in Eq. (2.2) of Ref. 14 and Eq. (4) above]. One thus finds, in the dirty metal limit $\left(l_{e} \ll W\right)$,

$$
\tau_{\Delta B}=12(\hbar / e \Delta B)^{2} / D W^{2},
$$

and, for a high-mobility channel $\left(l_{e} \gg W\right)$,

$\tau_{\Delta B}=4 C_{1}(\hbar / e \Delta B)^{2} / v_{F} W^{3}+2 C_{2}(\hbar / e \Delta B) l_{e} / v_{F} W^{2}$.

Equation (8) is a numerically obtained interpolation formula, the limits of small and large $\Delta B$ being exact. ${ }^{14}$ The coefficients $C_{1}, C_{2}$, and the diffusion coefficient $D$ depend on the type of boundary scattering. In the heterostructures considered the scattering is predominantly specular; ${ }^{15}$ then $C_{1} \approx 9.5, C_{2}=\frac{24}{5}$, and $D=\frac{1}{2} v_{F} l_{e}$. We stress that the results (7) and (8) are only valid for sufficiently small-field increments $\Delta B$, such that $\tau_{\Delta B} \gg \tau_{e}$, and $D \tau_{\Delta B} \gg W^{2}$. These inequalities are implied by the conditions $\tau_{\phi} \gg \tau_{e}, l_{\phi} \gg W$, mentioned earlier. The resulting restriction ${ }^{14}$ is that the flux increment $W^{2} \Delta B$ should be less than $\hbar / e$.

Upon substitution of Eq. (7) into Eq. (6), the criterion of Lee et al. ${ }^{11}$ for the dirty metal correlation field is recovered, ${ }^{19}$

$$
\Phi_{c} \equiv \Delta B_{c} W l_{\phi}(0)=\text { const } \times 2 \pi \hbar / e, \text { if } l_{e} \ll W .
$$

The numerical constant (left undetermined in Ref. 11) is given by $\left(3 / \pi^{2} \gamma\right)^{1 / 2}$, which equals 0.95 for $l_{\phi} \gg l_{T}$ and 0.42 for $l_{\phi} \ll l_{T}$. In a high-mobility channel, the "correlation flux" $\Phi_{c}$ resulting from Eqs. (6) and (8) is many times larger than the dirty metal value (9). For specular scattering we find

$$
\Phi_{c}=0.38 \gamma^{-1}(2 \pi \hbar / e) l_{e}^{2}\left[l_{\phi}(0) W\right]^{-1}\left\{1+\left[1+3.3 \gamma l_{\phi}(0)^{2} W l_{e}^{-3}\right]^{1 / 2}\right\} \text { if } l_{e} \gg W .
$$

In the regime $l_{\phi}(0) \gg l_{e} \sqrt{l_{e} / W}$, we see that $\Phi_{c}$ is larger than the dirty metal result (9) by a factor of order $\sqrt{l_{e} / W}$, while for $l_{e} \ll l_{\phi}(0) \lesssim l_{e} \sqrt{l_{e} / W}$ the enhancement factor can increase up to $l_{e} / W$. As a typical example, we have plotted in Fig. 1 $\Phi_{c}$ as a function of $l_{\phi}(0) / l_{e}$ for a channel with $l_{e}=5 \mathrm{~W}$, in the case $l_{\phi} \gg l_{T}$. For comparison, the constant dirty metal result is plotted as well. This figure illustrates the importance of the flux cancellation effect on magnetoresistance fluctuations in high-mobility channels. 
We have analyzed the UCF seen by van Houten et al. ${ }^{6}$ using the above results. The system studied is a narrow conducting channel, etched in the $2 \mathrm{D}$ electron gas of a GaAs- $\mathrm{Al}_{x} \mathrm{Ga}_{1-x} \mathrm{As}$ heterostructure. Estimates for the relevant lengths of the narrowest channel studied, at $T=2.4 \mathrm{~K}$, are $L=10 \mu \mathrm{m}, W=138 \mathrm{~nm}, l_{e}=314 \mathrm{~nm}$, $l_{T}=344 \mathrm{~nm}$. The conducting width $W$ of the channel is much smaller than the lithographic width of $0.5 \mu \mathrm{m}$, due to side wall depletion. The value of $W$ given above is obtained from an analysis ${ }^{6}$ of the magnetic depopulation of 1D subbands at high fields. An analysis of the low-field weak localization effect gives a value which is $20 \%$ lower, ${ }^{15}$ and comparable uncertainties exist in the values of $l_{e}$ and $l_{T}{ }^{20}$

In Fig. 2 we have plotted the correlation function $F(\Delta B)$ obtained from the magnetoresistance fluctuations shown in Fig. 3 of Ref. 6 . As usual, the impurity average in Eq. (1) is replaced by an average over $B$. A linear fit through the experimental data points was subtracted before calculating $F$, to correct for a systematic trend in $G(B)$. We find $F(0)=1.9 \times 10^{-4}\left(e^{2} / 2 \pi \hbar\right)^{2}, \Delta B_{c}=0.05$ $T$, with error estimates of $30 \%$. Substitution of this value for $F(0)$ into Eq. (3) gives a zero-field phase-coherence length $l_{\phi}(0)$ of $500 \mathrm{~nm}$. Equations (3), (5), and (8) then predict $\Delta B_{c}=0.12 \mathrm{~T}$, more than twice the experimental value. This discrepancy seems rather large to attribute entirely to uncertainties in $W$. More likely, the reason that the correlation field turns out smaller than predicted is that, as we increase the field increment, more and more electrons lose phase coherence before entering the regime of diffusive motion. This breakdown of coherent diffusion is beyond the UCF theory, which assumes $\tau_{\phi}(\Delta B) \gg \tau_{e}$,

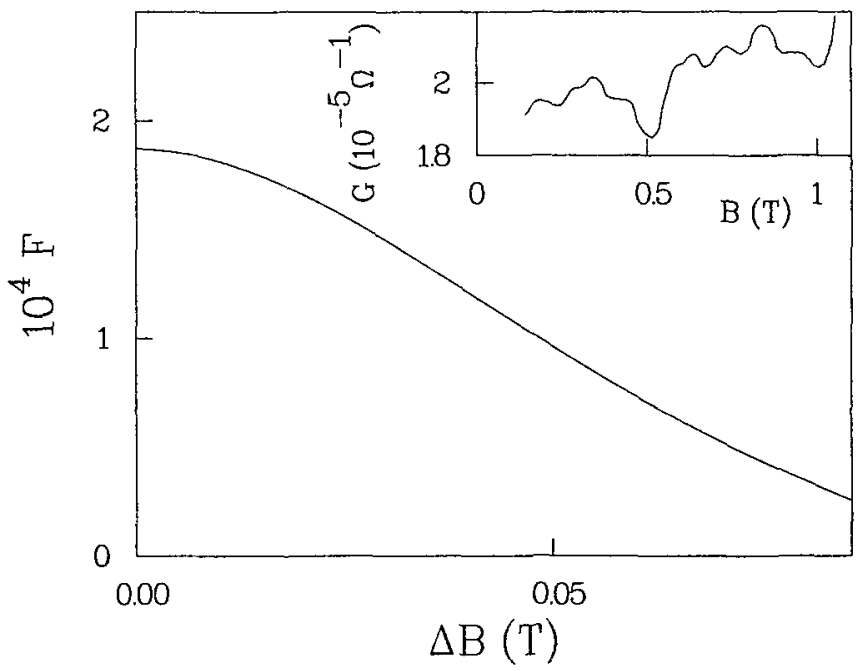

FIG. 2. Correlation function $F(\Delta B)$, in units of $\left(e^{2} / 2 \pi \hbar\right)^{2}$, obtained from the magnetoresistance measurements of Ref. 6. The inset shows the experimental data.

but certainly plays a role in systems where the phasecoherence time is comparable to the elastic scattering time $^{21}$ In the case above, the ratio $\tau_{\phi} / \tau_{e}$ is about 5 at zero field, which is not especially large (and becomes even smaller as we increase $\Delta B$ ). The situation in the similar experiment of Thornton et al. ${ }^{5}$ is even less favorable, with $\tau_{\phi} / \tau_{e}$ about 2 at the lowest temperature studied. The presented theory calls for experiments with a larger ratio of inelastic to elastic scattering times.
'B. L. Al'tshuler, Pis'ma Zh. Eksp. Teor. Fiz. 41, 530 (1985) [JETP Lett. 41, 648 (1985)].

2P. A. Lee and A. D. Stone, Phys. Rev. Lett. 55, 1622 (1985).

${ }^{3}$ S. Washburn and R. A. Webb, Adv. Phys. 35, 375 (1986).

${ }^{4}$ P. A. Lee, Physica A 140, 169 (1986).

${ }^{5}$ T. J. Thornton, M. Pepper, H. Ahmed, G. J. Davies, and D. Andrews, Phys. Rev. B 36, 4514 (1987)

${ }^{6}$ H. van Houten, B. J. van Wees, J. E. Mooij, G. Roos, and K.-F. Berggren, Superlattices Microstruct. 3, 497 (1988).

${ }^{7}$ R. P. Taylor et al., Surf. Sci. 196, 52 (1988).

${ }^{8}$ G. Timp et al., Phys. Rev. Lett. 59732 (1987); A. M. Chang et al., Superlattices Microstruct. (to be published).

${ }^{9}$ J. A. Simmons, D. C. Tsui, and G. Weimann, Surf. Sci. 196, 81 (1988).

${ }^{10}$ M. L. Roukes et al., Phys. Rev. Lett. 59, 3011 (1987).

"P. A. Lee, A. D. Stone, and H. Fukuyama, Phys. Rev. B 35, 1039 (1987).

12P. G. de Gennes and M. Tinkham, Physics (N.Y.) 1, 107 (1964); see also, P. G. de Gennes, Superconductivity of Metals and Alloys (Benjamin, New York, 1966), Chap. 8.

${ }^{13}$ V. K. Dugaev and D. E. Khmel'nitskii, Zh. Eksp. Teor. Fiz. 86, 1784 (1984) [Sov. 'Phys. JETP 59, 1038 (1984)].

${ }^{14} \mathrm{C}$. W. J. Beenakker and H. van Houten (unpublished).

${ }^{15} \mathrm{H}$. van Houten, C. W. J. Beenakker, B. J. van Wees, and J. E. Mooij, Surf. Sci. 196, 144 (1988).

${ }^{16}$ B. L. Al'tshuler and D. E. Khmel'nitskii, Pis'ma Zh. Eksp. Teor. Fiz. 42, 291 (1985) [JETP Lett. 42, 359 (1986)].

${ }^{17}$ S. Chakravarty and A. Schmid, Phys. Rep. 140, 193 (1986).
${ }^{18}$ The simple relation (4) holds (Ref. 14) only in the special gange $\Delta \mathbf{A}=\Delta B(z, 0,0)$, with the boundaries of the channel at $y=0, z= \pm W / 2$. For other choices of the vector potential the average phase factor depends on the initial and final coordinates of the trajectories, which would complicate a calculation. The ultimate result for the correlation function $F$ does not, of course, depend on the gauge.

${ }^{19}$ For completeness we also give the $T=0$ results: Lee et al. (Ref. 17) find $\Delta B_{c} W L=1.2(2 \pi \hbar / e)$, in the limit $l_{e} \ll W$. In the opposite limit $l_{e} \gg W$, we find that the correlation field is determined by the equation $D \tau_{\Delta B_{c}}=0.22 L^{2}$, with $\tau_{\Delta B_{c}}$ given by Eq. (8). For specular boundary scattering and in the regime $L \gg l_{e} \sqrt{l_{e} / W}$ this simplifies to $\Delta B_{c} W L=1.5(2 \pi \hbar /$ e) $\sqrt{l_{e} / W}$.

${ }^{20}$ The diffusion coefficient $D$, appearing in the definition of $l_{T}$, is determined from an estimate of the (classical) conductance at higher temperatures,

$$
G_{\mathrm{cl}}=\left(m e^{2} / \pi h^{2}\right)(W / L) D \approx 23 \times 10^{-6} \Omega^{-1},
$$

which gives $D=0.037 \mathrm{~m}^{2} \mathrm{~s}^{-1}$. The elastic length $l_{e}$ follows from the formula $D=\frac{1}{2} v_{F} l_{e}$, for specular boundary scattering. (The Fermi velocity $v_{F}=2.4 \times 10^{5} \mathrm{~ms}^{-1}$ results from the electron density obtained from the Shubnikov-de Haas oscillations).

${ }^{21}$ An ad hoc formula, which accounts to some extent for a finite $\tau_{\phi} / \tau_{e}$ ratio, has been used for the analysis of the weak localization effect in Ref. 15. 